\title{
CONGENITAL HEART DISEASE
}

\section{Idiopathic enlargement of the right atrium: 23 year follow up of a familial cluster and their unaffected relatives}

\author{
D J Kurz, E N Oechslin, R Kobza, R Jenni
}

Heart 2004;90:1310-1314. doi: 10.1136/hrt.2003.030023

See end of article for authors' affiliations .....................

Correspondence to: Professor R Jenni, Cardiology, University Hospital Zurich, Rämistrasse $100, \mathrm{CH}$ 8091 Zurich, Switzerland; karjer@usz.unizh.ch

Accepted 13 February 2004
Objective: To evaluate the long term outcome of familial idiopathic enlargement of the right atrium (IERA) and the risk of developing this disorder among unaffected offspring.

Design: 23 year follow up study.

Patients: 14 members (eight men, mean age 54 years, range $40-78$ ) of a pedigree with familial IERA. Methods: All patients were examined clinically and echocardiographically in 1979 and 2002. Normal cross sectional dimensions of the right atrium were derived from echocardiographic evaluation of 100 people $(47 \%$ men) with no structural or haemodynamic signs of heart disease. The 90th centile was chosen as the upper normal limit. IERA was defined as an increased right atrial long axis indexed to body surface area $\left(\right.$ RALAX ${ }_{i}$, men $>2.6 \mathrm{~cm} / \mathrm{m}^{2}$, women $\left.>2.8 \mathrm{~cm} / \mathrm{m}^{2}\right)$ in the absence of other cardiac abnormalities. Severe IERA was defined arbitrarily as RALAX $i$

Results: The course of the two index patients with severe IERA diagnosed in 1979 was complicated by atrial fibrillation, systemic embolism, and symptoms of heart failure without systolic dysfunction, resulting in the death of one man (77 years old). One of two patients with initially mild forms progressed to severe IERA. All of the initially unaffected offspring $(n=9)$ remained asymptomatic, although four of them had developed mild IERA.

Conclusions: During 23 years' follow up, severe IERA induced atrial fibrillation, systemic embolism, and symptoms of heart failure without systolic dysfunction in all cases in this family. Mild IERA seems to become manifest during middle age and may be followed by gradual progression to clinically relevant disease.
$\mathrm{T}$ he name "idiopathic enlargement of the right atrium" (IERA) was first coined by Pastor and Forte in their original description of this supposedly congenital malformation in $1961 .{ }^{1}$ Since then, more than 60 reports of this entity have been published, including a number of familial occurrences..$^{2-5}$ The diagnosis relies on the finding of a disproportionately enlarged right atrium in the absence of other cardiac or haemodynamic abnormalities ${ }^{6}$ and must be distinguished from other anomalies with a defined structural pathology of the right atrium, such as right atrial diverticula, aneurysms, and Ebstein's anomaly. ${ }^{28}$ IERA has been reported to occur at all stages of life, in most cases being discovered incidentally, with reports of presentation age ranging from the intrauterine fetus ${ }^{8}$ to the elderly. ${ }^{10}$ However, very little is known about the natural history and long term prognosis of this disorder.

In 1979 we described two siblings with IERA and screened their first degree relatives for the presence of this anomaly, finding two further cases. ${ }^{3}$ Here we report the 23 year follow up of this cluster and their initially unaffected relatives. Issues of interest were as follows. Which complications occur during long term follow up of IERA? Do patients with mild forms progress to severe IERA? Will unaffected offspring of IERA patients develop this disorder with ongoing age?

\section{METHODS}

\section{Patients and measurements}

All family members investigated in 1979 were invited to a follow up examination. One sister of the parent generation requiring care in a nursing home and three members of the offspring generation currently living abroad were unavailable for follow up. For the remaining 14 family members, a medical history since the initial examination was taken and verified with the primary care physicians. A physical examination, a 12 lead ECG, a complete echocardiographic study, and-where appropriate-a frontal and lateral chest radiograph were obtained from each subject. The cardiothoracic ratio was defined as the largest transverse diameter of the heart silhouette divided by that of the inside of the bony thorax in the posteroanterior chest radiograph, with normal values considered to be less than $0.55 .{ }^{11}$

\section{Echocardiographic measurements}

Transthoracic Doppler echocardiography was performed in standard imaging planes. The long and short axis of the right atrium were measured at the end of ventricular systole in the apical four chamber view. The right atrial long axis (RALAX) was defined as the distance from the hinge point of the septal tricuspid valve leaflet to the roof of the right atrium, measured along a line parallel to the interatrial septum. The short axis was defined as the distance between the interatrial septum and the right atrial free wall, with the axis cutting the RALAX at right angles and at mid length. Measurements were adjusted to body surface area (BSA), which was calculated by the DuBois formula: BSA = $0.00718 \times[\text { weight in } \mathrm{kg}]^{0.425} \times[\text { height in } \mathrm{cm}]^{0.725} .{ }^{12}$

\section{Statistical analysis}

The unpaired, two tailed $t$ test was used to compare continuous variables. Values of $\mathrm{p}<0.05$ were considered significant.

Abbreviations: BSA, body surface area; IERA, idiopathic enlargement of the right atrium; RALAX, right atrial long axis; RALAX ${ }_{i}$, right atrial long axis indexed to body surface area 
RESULTS

\section{Determination of normal right atrial size and} definition of IERA

To identify patients with mild forms of right atrial enlargement, it was necessary to define the normal echocardiographic dimensions of this chamber in a central European population. To achieve this, we retrospectively analysed routine echocardiography studies performed at our institute on 100 patients (47\% men) with no structural or haemodynamic abnormalities. The majority of these patients were either being evaluated as potential live organ transplant donors or were having their left ventricular function assessed before first time treatment with cardiotoxic chemotherapeutic agents. The age distribution of these patients was similar between sexes, with the mean (SD) age being 42.9 (15.9) years for men and 42.1 ( 13.5$)$ years for women $(\mathrm{p}=0.8)$. Figure 1 shows the distribution of right atrial sizes. We found that the RALAX indexed to the BSA $\left(\right.$ RALAX $\left._{\mathrm{i}}\right)$, was significantly larger in women than in men (mean (SD) 2.56 $\left.(0.24) \mathrm{cm} / \mathrm{m}^{2} v 2.34(0.23) \mathrm{cm} / \mathrm{m}^{2}, \mathrm{p}<0.0001\right)$. Although the short axis also tended to be larger in women (mean (SD) $\left.1.94(0.27) \mathrm{cm} / \mathrm{m}^{2} v 1.84(0.29) \mathrm{cm} / \mathrm{m}^{2}\right)$, this was not significant. On account of this and the better reproducibility of the long axis compared with the short axis, we decided to assess right atrial size based on the RALAX $X_{\mathrm{i}}$. We defined the 90th centile as the upper limit of normal (men $2.6 \mathrm{~cm} / \mathrm{m}^{2}$, women $2.8 \mathrm{~cm} / \mathrm{m}^{2}$ ). Apart from the sex difference, these values are consistent with two previous publications evaluating the normal range of right atrial dimensions. ${ }^{13} 14$

On the basis of these values we defined IERA as an increased RALAX $_{\mathrm{i}}$ in the absence of other structural or haemodynamic cardiac abnormalities. Severe IERA was arbitrarily defined to be present if $\operatorname{RALAX}_{\mathrm{i}} \geqslant 4 \mathrm{~cm} / \mathrm{m}^{2}$ and mild IERA was defined as RALAX ${ }_{i}$ larger than the upper limit of normal but $<4 \mathrm{~cm} / \mathrm{m}^{2}$.

\section{Findings at 23 years' follow up}

In 1979 we diagnosed striking examples of IERA in two siblings aged 58 and 55 years. At the time, both were being evaluated for chronic atrial fibrillation with mild exertional dyspnoea and cardiomegaly on the chest radiograph. ${ }^{3}$ Their further course was in each case complicated by symptoms of heart failure with an onset after a lag phase of many years and the occurrence of systemic embolism in the absence of anticoagulant treatment. In patient 1, stroke and heart failure led to death in a nursing home at the age of 77 years. However, his course had been stable and relatively free of symptoms until only a few months previously. His sister, now 78 years of age (patient 2, fig $2 \mathrm{~A}-\mathrm{C}$ ) had recently developed progressive exertional dyspnoea but was now doing well on a regimen of angiotensin converting enzyme inhibitors, diuretics, and digoxin. Echocardiography showed normal size and function of both ventricles (fig 2C). The size of the giant right atrium was similar to its size in 1979. It had caused annular dilatation of the tricuspid valve (end systolic diameter in the four chamber view $3.2 \mathrm{~cm}$ ), leading to moderate tricuspid regurgitation (not present in 1979). Moderate pulmonary hypertension, which had initially not been present, had developed during follow up (in 2002 the peak systolic tricuspid valve gradient was $43 \mathrm{~mm} \mathrm{Hg}$; in 1979 invasively measured pulmonary artery pressure was $27 / 10 \mathrm{~mm} \mathrm{Hg}$ and pulmonary vascular resistance 57 dynes.s. $\mathrm{cm}^{-5}$ ). In 1997 arterial embolism to the leg had occurred. At that point in time, an oral anticoagulant had not yet been introduced to her regimen, despite the presence of atrial fibrillation. After subsequent initiation of anticoagulation, no further episodes of systemic embolism were observed.

Of the two relatives with initially mild forms, another male sibling (patient 3, fig 2 D-F), now aged 76 years, had
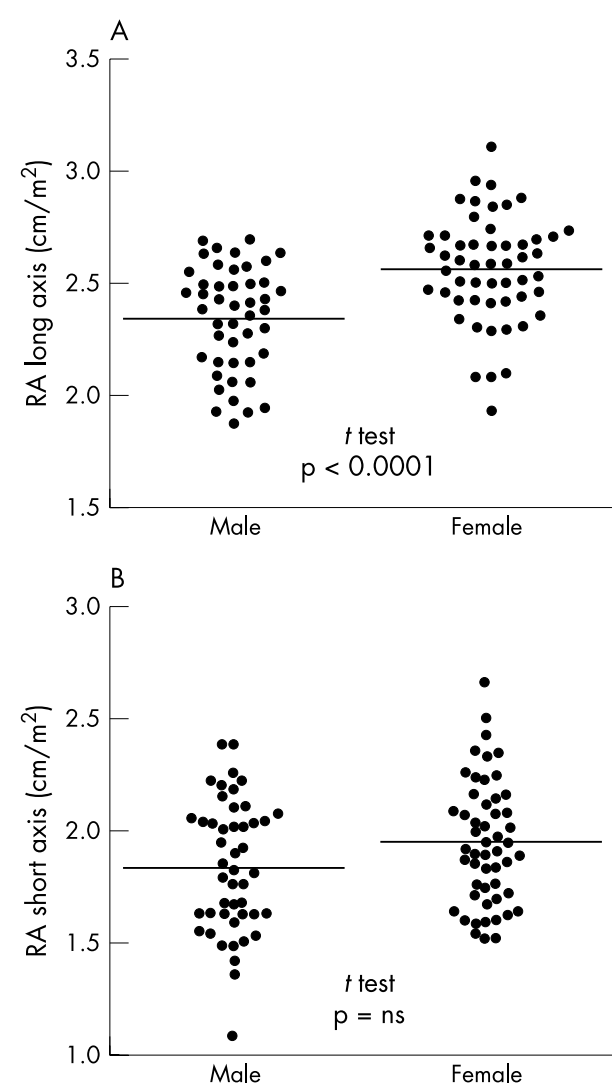

Figure 1 Distribution of individual (black circles) and mean (horizontal bar) right atrial (RA) long axis (A) and short axis (B) dimensions among 100 patients $(47 \%$ men) with no structural or haemodynamic abnormalities. Values are corrected for body surface area.

progressed to severe IERA. He had recently developed mild exertional dyspnoea and had atrial fibrillation diagnosed. In 1999 an oral anticoagulant was introduced after a transient ischaemic attack, with no subsequent recurrences. As in the previous case, dimensions and systolic function of both ventricles were normal. His RALAX ${ }_{i}$, which in 1979 had been $3.1 \mathrm{~cm} / \mathrm{m}^{2}$, now measured $4.7 \mathrm{~cm} / \mathrm{m}^{2}$. Tricuspid regurgitation was mild. The peak systolic pressure gradient over the tricuspid valve of $27 \mathrm{~mm} \mathrm{Hg}$ indicated mildly increased pulmonary pressures. In summary, by the time of follow up, severe symptomatic IERA was diagnosed in three of five siblings of the parent generation, one of whom had died of disease related complications 19 years after the diagnosis.

Among the offspring generation available for follow up $(n=10)$, the eldest son of patient 1 had already had mild IERA in 1979, which had not progressed since then (1979 RALAX $_{\mathrm{i}} 3.1 \mathrm{~cm} / \mathrm{m}^{2} v 3.0 \mathrm{~cm} / \mathrm{m}^{2}$ in 2002). Of the nine initially unaffected children, all had remained asymptomatic and in sinus rhythm. They were now between 40 and 51 years old (mean (SD) 46 (4) years). However, in four of them (44\%) echocardiography showed the development of mild right atrial enlargement $\left(\right.$ RALAX $_{\mathrm{i}}$ range $\left.2.7-3.2 \mathrm{~cm} / \mathrm{m}^{2}\right)$. In all cases, echocardiographic studies were otherwise unremarkable, showing normal dimensions and function of both ventricles, no enlargement of the left atrium, and no pulmonary hypertension. Figure 3 summarises the disease state of the individual members of this pedigree and their progression during long term follow up.

\section{DISCUSSION}

Despite a large number of publications describing cases of IERA, surprisingly little is known about the natural history of 
1979
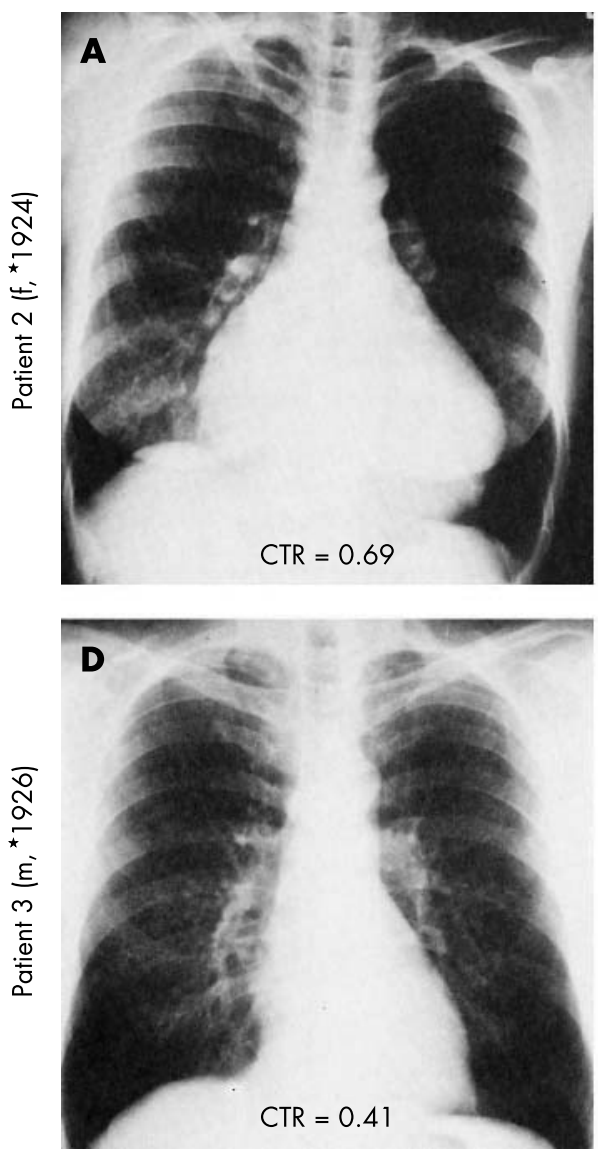

2002
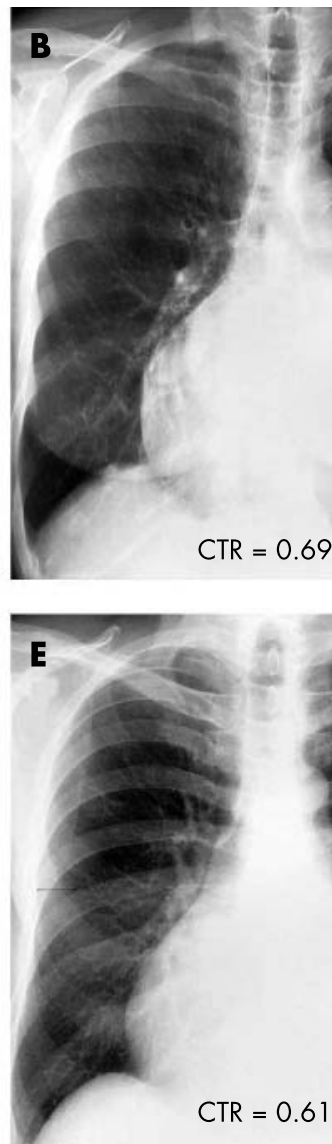

2002
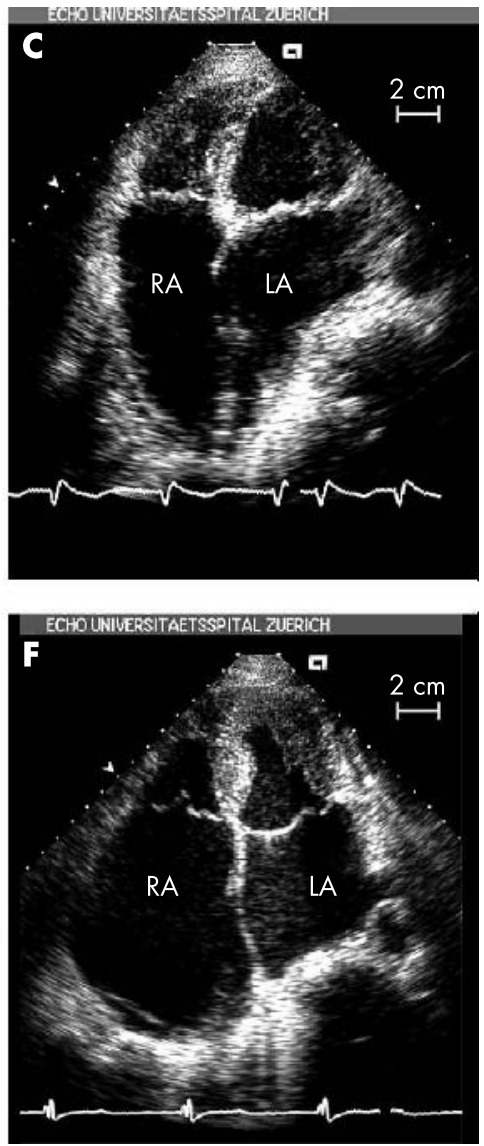

Figure 2 Chest radiographs and echocardiograms of two siblings with idiopathic enlargement of the right atrium (IERA). Patient 2 had severe IERA in 1979 (A) with similar findings in 2002 (B, C). Patient 3 had mild IERA in 1979 (D) but had progressed to severe disease by 2002 (E, F). Note the normal dimensions of both ventricles in the apical four chamber view (C, F). CTR, cardiothoracic ratio. RA, right atrium. LA, left atrium.

this anomaly. In most published cases, follow up data are not available. In this long term follow up study, we observed the occurrence of atrial fibrillation, systemic embolism, and symptoms of heart failure in all three cases of severe IERA. In the two of these patients available for echocardiographic follow up (patients 2 and 3), the cause of exertional dyspnoea was not immediately evident. Dimensions and systolic function of both ventricles were normal but diastolic dysfunction could not be reliably assessed due to the presence of atrial fibrillation. Dyspnoea may have been caused, at least in part, by the reduction of cardiac output known to result from the loss of atrial function during atrial fibrillation, with symptoms further being augmented by the increased pulmonary artery pressures that had developed during follow up. In the absence of overt pulmonary or left sided heart disease, one possible cause of pulmonary hypertension in these patients may have been recurrent pulmonary embolism. Both patients were in atrial fibrillation for many years before the initiation of anticoagulant treatment and in this setting one can readily imagine the fibrillating giant right atrium as a source of thromboembolic events. In this context, it was noteworthy that systemic embolism was a complication observed in all three siblings with severe IERA. Each of these episodes occurred in the absence of anticoagulant treatment and subsequent oral anticoagulation was successful in preventing further ischaemic events in patients 2 and 3 . The course of these patients is in accordance with current understanding that patients with large atria have a very high risk of thromboembolic events after the onset of atrial fibrillation. These two cases illustrate the importance of early and strict long term anticoagulant treatment of patients with IERA with this complication.

Similar outcomes have been reported in a series of eight elderly patients from Japan (mean age 70 years, follow up 5-12 years) with idiopathic biatrial dilatation. ${ }^{10}$ In these patients atrial fibrillation and symptoms of heart failure were universally present and response to medical treatment with diuretics and digitalis was satisfactory. Other complications reported to have occurred in IERA, but which we did not observe, are supraventricular arrhythmias other than atrial fibrillation, atrioventricular conduction block, and in two cases sudden cardiac death. ${ }^{2}{ }^{15}$ Our findings support previous reports indicating that in the vast majority of cases IERA is a relatively benign disorder, which in general can be managed with anticoagulant and medical treatment, with surgical procedures being reserved for patients with mechanical complications or uncontrolled symptomatic arrhythmia.

Reports of familial occurrences of IERA are rare. This may be related to the fact that the diagnosis is often made by chance at an advanced age, a situation in which other family members may not usually be examined. An important aspect of this study is therefore the echocardiographic documentation of the natural history of this disorder in first degree relatives with initially mild forms or even absence of IERA. As illustrated by patient 3, mild cases can progress with advancing age to severe IERA, with all the typically associated symptoms. Furthermore, it was noteworthy that four of nine $(44 \%)$ members of the offspring generation, who at the time of the original examination in 1979 had normal echocardiographic studies, had gone on to develop mild IERA 
A

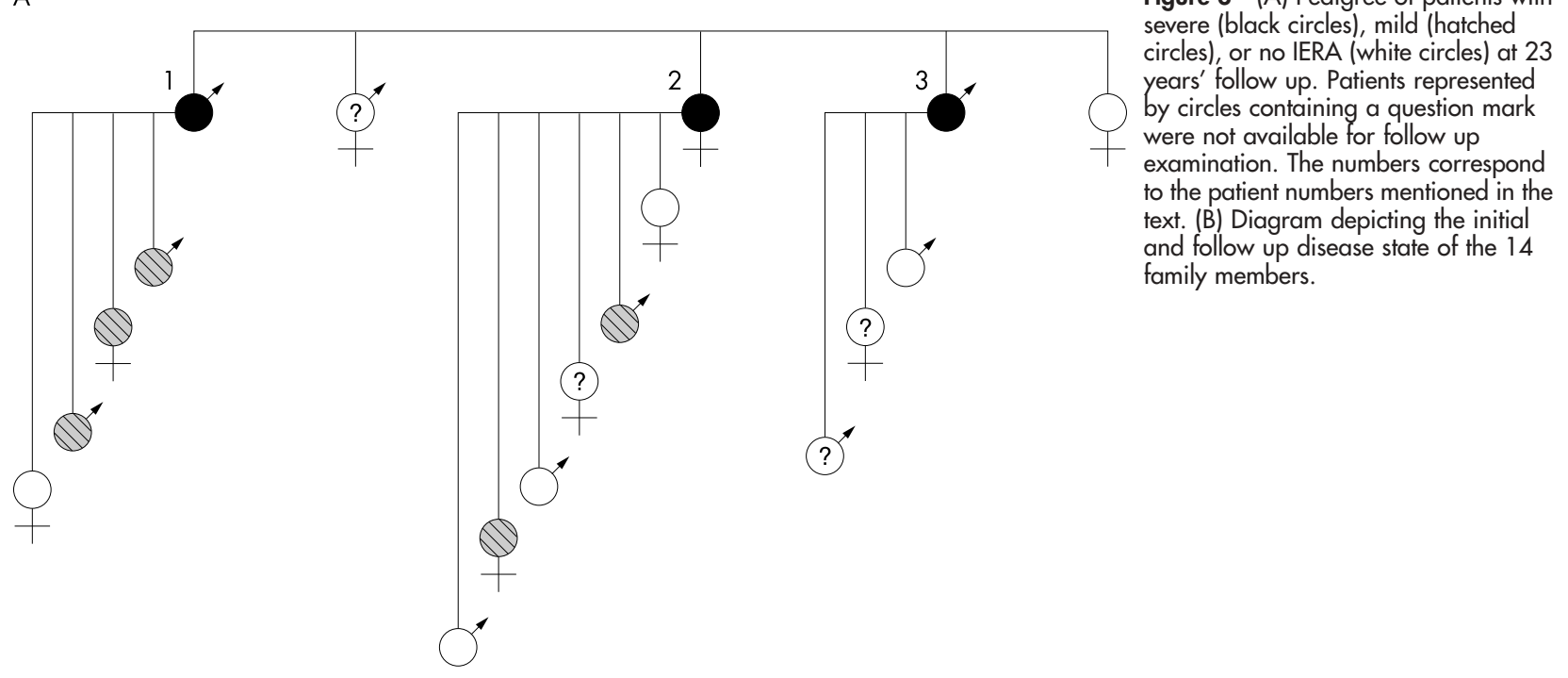

Figure 3 (A) Pedigree of patients with severe (black circles), mild (hatched circles), or no IERA (white circles) at 23 years' follow up. Patients represented by circles containing a question mark were not available for follow up examination. The numbers correspond to the patient numbers mentioned in the (B) Diagram depicting the initial and follow up disease state of the 14 amily members.

B

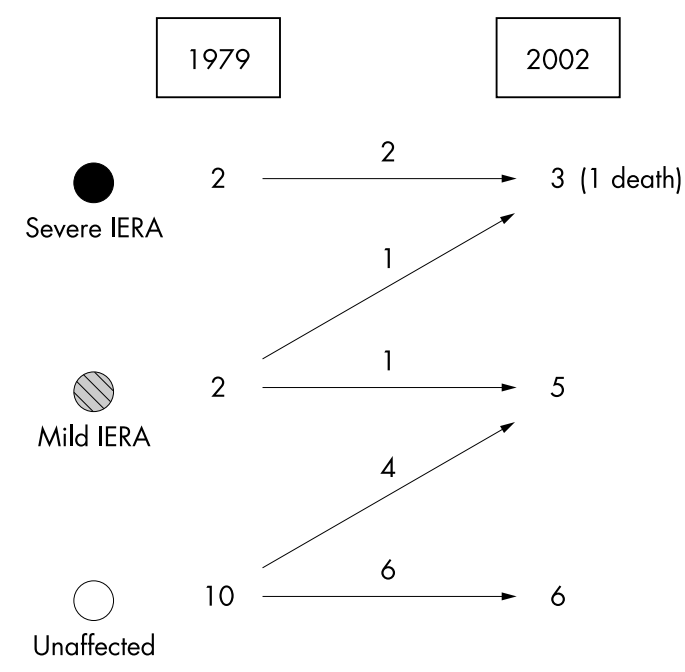

during early middle age. All of these patients were still without symptoms or atrial arrhythmias at follow up.

Since dilatation of the right atrium in the offspring generation generally appeared mild, confirmation was necessary that dilatation was present compared with a healthy central European population. We therefore determined normal dimensions of the right atrium in 100 people with no structural or haemodynamic signs of heart disease (fig 1) and defined the upper limit of normal as the 90th centile of the right atrial long axis, indexed to the BSA. Previously published literature on the normal dimensions of the right atrium are sparse but are comparable with our findings. ${ }^{13}{ }^{14}$ In the past, published values were not corrected for BSA, a biometric ploy that we found to be valuable in limiting the spread of measured values. Furthermore, a sex difference in the dimension of the right atrium has not been described previously. In our series, this difference was present in both axes but was significant only for the long axis.

The distribution of cases among consecutive generations suggests a genetic mechanism for IERA. The finding that three of five siblings of the parent generation and five of 10 direct offspring are affected makes an autosomal dominant defect seem likely. One may speculate that mild forms are a "forme fruste" or decreased penetration of the severe disorder. Whether the offspring who now have mild IERA will progress to severe dilatation with advancing age remains to be seen. Unfortunately, the number of members in the pedigree presented here was too small to allow a search for candidate genes responsible for IERA with chromosomal mapping techniques.

The wide range of ages at which IERA has been reported to become clinically manifest suggests that IERA may be a phenotypic syndrome rather than a unique disease entity. It seems unlikely that the form of IERA developing in middle age, as found in this pedigree and which also corresponds to the majority of cases described in the literature, is the same disorder as the congenital anomaly present at birth. This concept of IERA as a clinical syndrome rather than a nosological entity would also accommodate the different spectrum of arrhythmic disturbances associated with IERA of paediatric or middle age onset. ${ }^{2} 1015$

In conclusion, this study shows that patients with severe IERA can do well over decades under medical treatment, despite a high prevalence of atrial fibrillation and symptoms of heart failure without systolic dysfunction. This form of IERA seems to become manifest initially during middle age 
and may be followed by gradual progression to clinically relevant disease. Considering the high frequency of systemic embolism among our patients with severe IERA, early initiation of long term anticoagulant treatment must be recommended upon the onset of atrial fibrillation. It therefore appears appropriate to monitor relatives affected with mild IERA at regular intervals for the development of this complication.

\section{ACKNOWLEDGEMENTS}

David J Kurz receives financial support from the Swiss National Science Foundation and the Swiss Heart Foundation.

\section{Authors' affiliations \\ D J Kurz, E N Oechslin, R Kobza, R Jenni, Echocardiography, Cardiovascular Centre, University Hospital, Zurich, Switzerland}

\section{REFERENCES}

1 Pastor BH, Forte AL. Idiopathic enlargement of the right atrium. Am J Cardiol $1961 ; 8: 513-8$.

2 Binder TM, Rosenheck R, Frank $\mathrm{H}$, et al. Congenital malformations of the right atrium and coronary sinus. Chest 2000;117:1740-8.

3 Jenni R, Goebel N, Schneider L, et al. [Idiopathic familial right atrial dilatation]. Schweiz Med Wochenschr 1981;111:1565-72.
4 Takahashi M, Nakagawa Y, Ogawa N, et al. [Familial idiopathic enlargement of the right atrium]. Kokyu To Junkan 1988;36:573-6.

5 Blondheim DS, Klein R, Plich M, et al. Familial idiopathic dilatation of the right atrium with complete atrio-ventricular block: a new syndrome? Cardiology 2000:94:224-6.

6 Sumner RG, Phillips JH, Jacoby WJ Jr, et al. Idiopathic enlargement of the right atrium. Circulation 1965;32:985-91.

7 Kobza R, Oechslin E, Pretre R, et al. Enlargement of the right atrium: diverticulum or aneurysm. Eur J Echocardiogr 2003:4:223-5.

8 Reinhardt-Owlya L, Sekarski N, Hurni M, et al. [Idiopathic dilatation of the right atrium simulating Ebstein's anomaly: apropos of a case diagnosed in utero]. Arch Mal Coeur Vaiss 1998:91:645-9.

9 Asayama J, Matsuura T, Endo N, et al. Idiopathic enlargement of the right atrium. Am J Cardiol 1977;40:620-3.

10 Arima M, Kanoh T, Okazaki S, et al. Clinical manifestation and survival of patients with idiopathic bilateral atrial dilatation. Intern Med 1999;38:1 12-8.

11 Kabala JE, Wilde P. Measurement of heart size in the anteroposterior chest radiograph. Br J Radiol 1987;60:981-6.

12 DuBois D, DuBois EF. A formula to estimate the approximate surface area if height and weight be known. Arch Intern Med 1916;17:863-71.

13 Bommer W, Weinert L, Neumann A, et al. Determination of right atrial and right ventricular size by two-dimensional echocardiography. Circulation 1979;60:91-100.

14 Anon. Appendix A. Normal cross-sectional echocardiographic measurements. In: Weymann AE, ed. Principles and practice of echocardiography. 2nd ed. Philadelphia: Lippincott Williams and Wilkins, 1994: 1289-98.

15 Blaysat G, Villain E, Marcon F, et al. [Prognosis and outcome of idiopathic dilatation of the right atrium in children: a cooperative study of 15 cases]. Arch Mal Coeur Vaiss 1997;90:645-8.

\section{IMAGES IN CARDIOLOGY}

\section{A 38 year old well functioning Starr-Edwards valve prosthesis}

doi: 10.1136/hrt.2004.035741

A 74 year old man with Canadian Cardiovascular Society class II stable angina was referred to São Lucas Hospital, Porto Alegre, Brazil, for cardiac catheterisation in May 2003. He had a past medical history significant for aortic valve stenosis, having previously been treated in August 1965 with a Starr-Edwards metallic valve prosthesis; the surgery was performed at the University of Indiana Hospital, Indianapolis, USA.

Coronary angiography revealed a moderate proximal circumflex artery lesion. In the fluoroscopic evaluation, the caged ball prosthesis presented normal mobility and no signs of structural deterioration (panels A and B). Ascendant aortography showed a $5.5 \mathrm{~cm}$ aneurysm and mild aortic regurgitation. Transoesophageal echocardiography showed an ascendant aortic aneurysm, a well functioning aortic valve prosthesis with minimal regurgitation, and a turbulent systolic jet with a mean pressure gradient of $18 \mathrm{~mm} \mathrm{Hg}$. Left ventricular ejection fraction was estimated at $60 \%$. The patient has been on anticoagulation since the surgery with no significant clinical complication. Since the coronary

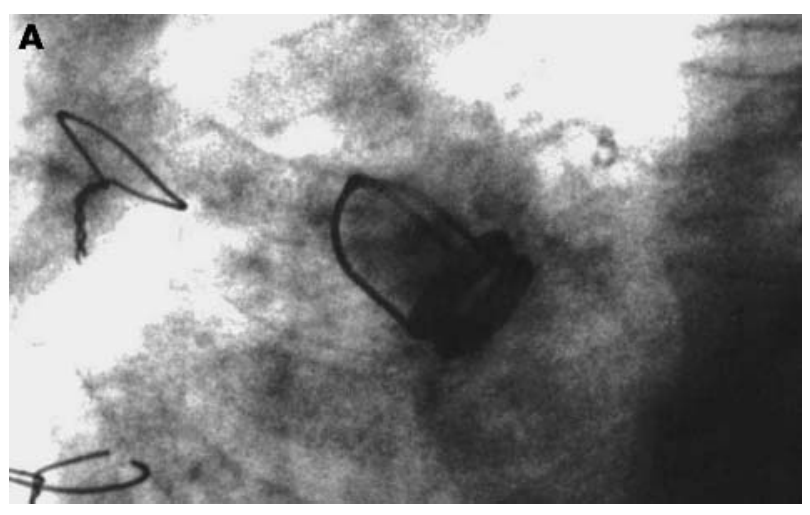

angiogram, the patient has been kept on medical treatment for coronary artery disease, and remains in stable condition.

The first case of aortic valve replacement with the StarrEdwards metallic prosthesis was reported in 1965. This prosthesis was also used to replace the mitral valve. However, the mitral replacement with this prosthesis was abandoned because of thrombotic complications. In the aortic position, the Starr-Edwards metallic valve was widely used until the 1970s, when it started to be replaced by the tilting disc metallic valves. This case report demonstrates the impressive durability of the Starr-Edwards prosthesis in the aortic position.

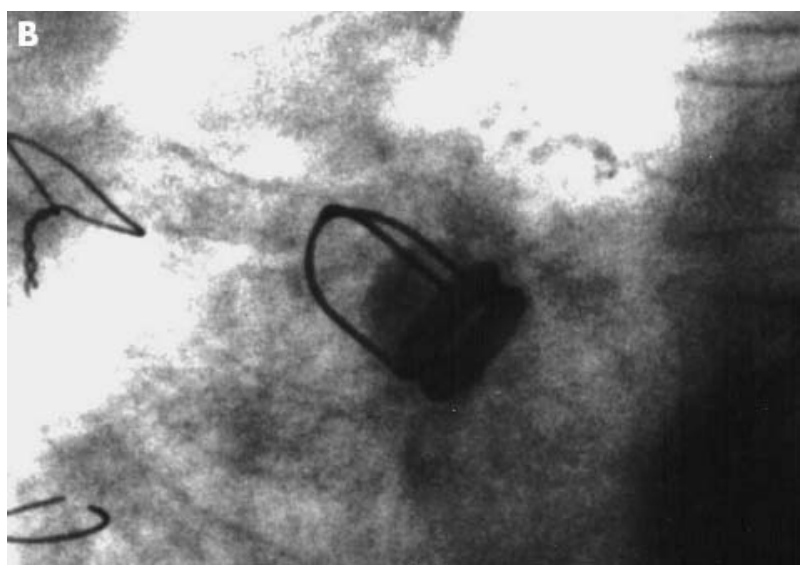

V O Gomes

A A Brizolara

R Lasevitch

P R A Caramori

caramori.p@plug-in.com.br 